\title{
Going paperless: improved cataract surgery outcome data quality in a new fully electronic unit
}

\author{
A. Z. Nghiem $\mathbb{D}^{1}$ - C. Canning ${ }^{1} \cdot$ J. Eason ${ }^{1}$ - J. M. Sparrow ${ }^{2,3} \cdot$ T. H. Flynn ${ }^{1}$
}

Received: 11 March 2018 / Revised: 10 October 2018 / Accepted: 7 November 2018 / Published online: 11 February 2019

(c) The Royal College of Ophthalmologists 2019

\begin{abstract}
Objectives To report outcome data on the first 5000 consecutive cataract cases at a new paperless eye unit and benchmark against the Royal College of Ophthalmologists' National Ophthalmology Database (RCOphth NOD).

Methods Using the in-built audit tool of the electronic medical records system, data from all cataract operations performed between 1 April 2014 and 13 January 2017 were compiled.

Results Five thousand and eight cases were recorded of which the overall intra-operative complication rate was $2.4 \%$, the most common being posterior capsular rupture-1.14\%. Follow-up data on post-operative complications were recorded in $98.6 \%$ of cases. Pre- and post-operative visual acuities was measured in $98.0 \%$ of cases. In all, $40.8 \%$ of eyes achieved a visual acuity of $6 / 6$ or better and $90.7 \%$ achieved $6 / 12$ or better.

Conclusions A data set of $>5000$ consecutive cataract operations was obtained in this eye department. The recording of preand post-operative visual acuity in $98 \%$ of cases compare very favourably to the RCOphth NOD Audit Report 2017 where pre- and post-operative visual acuities were recorded in only $57.1 \%$ of operations. Despite this difference, the outcome measures from this unit and RCOphth NOD were very similar, validating the results of the RCOphth NOD audit reports. Significantly, when applying the RCOphth NOD audit criteria for measuring post-operative visual acuity, approximately $15 \%$ of cases were excluded from the data set, reducing the completeness of the data set. Paperless ophthalmology units are feasible in today's NHS and can produce near complete cataract data sets; this can ultimately lead to more comprehensive and reliable aggregate cataract outcome data.
\end{abstract}

\section{Introduction}

Cataract surgery is the most common surgical procedure in the UK, where 330,000 cataract operations are performed per year in the English National Health Service (NHS) in the UK [1]. In recent years, there has been increasing emphasis on publication of aggregate and individual surgical outcome data in cataract surgery [2]. Publication of surgical outcomes is an important driver of quality

T. H. Flynn

Tom.Flynn@nhs.net

1 Moorfields Eye Centre at Croydon University Hospital, 530 London Road, Thornton Heath, Surrey CR7 7YE, UK

2 Bristol Eye Hospital, Lower Maudlin Street, Bristol BS1 2LX, UK

3 Population Health Sciences, Bristol Medical School, University of Bristol, Canynge Hall, 39 Whatley Road, Bristol BS8 2PS, UK improvement and helps patients to make informed decisions about their care.

The primary dimensions of data quality have been defined as completeness, uniqueness, timeliness, validity, accuracy and consistency [3]. In many reports on cataract outcomes to date, there appears to be actual or potential data quality issues both in terms of the accuracy (representativeness) of the data and the completeness of the data set. Although some databases have been able to capture a large number of operations, the results have been limited by incomplete collection of key primary outcome indicators, which in turn may affect the quality of the data. In addition, when outcome databases are dependent on input of data that is separate from the clinical record there is selection bias and potential loss of representativeness of the data.

The European Registry of Quality Outcomes for Cataract and Refractive Surgery (EUREQUO) reported outcomes on 523,921 cataract extractions but long-term follow-up data (760 days) were available in only $46 \%$ of cases [4]. The Royal College of Ophthalmologists' National Ophthalmology 
Database (RCOphth NOD) aims to provide robust evidence on cataract surgery outcomes and in its first report has audited the outcomes of 75,827 cataract operations in 34 centres in England [2]. However, the results drawn from this first report indicate that data on pre- and post-operative visual acuity (VA) were recorded in the database in only $52.7 \%$ of cases. In the second RCOphth NOD audit report in 2017, this figure improved to $57.1 \%$ of cases [5]. An estimate of the proportion of cataract operations performed in each participating centre that was included in the RCOphth NOD audit report ranged from 7.7 to $99.9 \%$ (overall $73 \%$ ).

In the case of RCOphth NOD, the incompleteness of the data set is due partly to the time-based definition of postoperative VA (only cases with an acuity measured between 2 weeks to 4 months post-surgery are included). A more important factor is the fact that, although many ophthalmology units in the UK use electronic medical records (EMR), very few use EMR exclusively. The ongoing partial use of paper records is the main reason for data leaching from multicentre electronic data sets such as the RCOphth NOD through the patients' cataract pathways. The NHS in England plans to be paperless by 2023 [6]. In 2014, Moorfields Eye Hospital NHS Foundation Trust established a new paperless ophthalmology unit at Croydon University Hospital. We present outcomes data on the first 5008 consecutive cataract surgery cases performed at this new unit.

Our specific aims were:

1. To benchmark our cataract surgery results against the RCOphth NOD results using the RCOphth NOD definition of post-operative VA in order to assess the representativeness of our data.

2. To investigate whether including data from patients seen and discharged within 2 weeks of surgery made a material difference to the visual outcomes.

3. To report outcomes on this more inclusive and almost complete data set of 5008 consecutive cases.

\section{Methods}

Moorfields Eye Centre at Croydon University Hospital uses a single EMR system to record cataract encounters (Medisoft Ophthalmology, Medisoft Limited, Leeds, UK).

All cataract operations were performed between 1 April 2014 and 13 January 2017. These dates represent the opening date of the new eye unit and the date at which the 5000 th cataract operation was performed.

All duplicate records and records not belonging to patients (e.g. test patients) were removed by Medisoft technical staff. Thereafter, the in-built audit tool in the EMR was used to acquire data. The search was conducted on the 12 December 2017.

Baseline data on demographics, pre-operative VA, ocular co-morbidities and whether the surgery was on a first or second eye were collected. Outcome data on intra-operative complications, post-operative complications, post-operative VA and deviation from predicted post-operative refraction were collected. Pre-operative VA data was defined as the better value of uncorrected distance visual acuity (UDVA) or corrected distance visual acuity (CDVA). Post-operative 'best-measured' VA was defined as the best CDVA measurement when present and the best measurement of UDVA or pinhole VA when CDVA was absent. Post-operative VA data were acquired in two ways: first, using the RCOphth NOD timescale of 2 weeks to 4 months post-surgery and, second, using a more inclusive timescale of 1 day to 6 months post-surgery.

\section{Results}

Five thousand and eight cataract operations were recorded between 1 April 2014 and 13 January 2017 at Moorfields Eye Centre at Croydon University Hospital. Two thousand nine hundred and two (57.9\%) were female and 2106 $(42.1 \%)$ were male and the mean age was 73.6 years. In all, $41.2 \%$ of operations were performed by consultants, $38.6 \%$ by career grade non-consultant surgeons, $15.5 \%$ by experienced trainees and $4.7 \%$ by less experienced trainees.

The presence or absence of ocular co-pathology was documented in $100 \%$ of cases. Three thousand five hundred and nineteen $(70.3 \%)$ operations were in patients with no recorded ocular co-pathology and 1489 (29.7\%) were in patients with recorded ocular co-pathology.

\section{Intra-operative complications}

The intra-operative complication rate was $2.4 \%$ (119 cases), the most common being posterior capsular rupture (PCR) which occurred in $1.14 \%$ of cases (see Table 1). The RCOphth NOD uses the definition of PCR to include PCR with and without vitreous loss and zonular rupture with vitreous loss.

\section{Post-operative complications}

Follow-up data on post-operative complications were recorded in $4938(98.6 \%)$ of operated eyes. The overall post-operative complication rate was $9.8 \%$, the most common being post-operative uveitis (129 cases, 2.6\%) and cystoid macular oedema (99 cases, 2.0\%), see Table 2 . 
Table 1 Intra-operative complications compared to RCOphth NOD 2017

\begin{tabular}{lcl}
\hline Intra-operative complications & $n(\%)$ & $\begin{array}{l}\text { RCOphth NOD } \\
2017, \%\end{array}$ \\
\hline $\begin{array}{l}\text { No intra-operative } \\
\text { complications }\end{array}$ & $4889(97.6)$ & 96.7 \\
One or more intra-operative & $119(2.4)$ & 3.3 \\
complications & & \\
PCR $^{\mathrm{a}}$ & $57^{\mathrm{a}}(1.14)$ & $1.5^{\mathrm{a}}$ \\
Corneal epithelial abrasion & $13(0.3)$ & 0.3 \\
Zonule dialysis & $10(0.2)$ & 0.4 \\
Endothelial damage/Descemet's & $7(0.1)$ & 0.1 \\
tear & $6(0.1)$ & $<0.1$ \\
Phaco burn/wound problems & $3(0.1)$ & 0.2 \\
Torn iris/damage from phaco & $2(<0.1)$ & $<0.1$ \\
Hyphaema & $1(<0.1)$ & 0.1 \\
IOL exchange & $1(<0.1)$ & $<0.1$ \\
Iris prolapsed & $1(<0.1)$ & - \\
Operation cancelled & $1(<0.1)$ & 0.1 \\
Other IOL problem & $25(0.5)$ & 0.4 \\
Other &
\end{tabular}

$I O L$ intraocular lens, $P C R$ posterior capsular rupture, RCOphth NOD Royal College of Ophthalmologists' National Ophthalmology Database

${ }^{a} \mathrm{PCR}$ figure includes zonule rupture with vitreous loss and lens fragment into vitreous

\section{Visual acuity}

The pre-operative VA was recorded in 4927 (98.4\%) out of 5008 cases.

Overall, 4906 eyes (98\%) had documented VA before and after cataract surgery. Using the RCOphth NOD time criteria of measuring post-operative VA between 2 weeks and 4 months post-surgery, 4156 (83\%) eyes had documented VA before and after cataract surgery. In all, $15 \%$ of cases were reviewed and discharged within 2 weeks of surgery.

Overall, $2004(40.8 \%)$ of patients achieved a postoperative VA of 6/6 or better and 4449 (90.7\%) achieved 6/ 12 or better after surgery (see Table 3 ). There was broad agreement in visual outcomes between our comprehensive data, our data limited to RCOphth NOD time criteria for post-operative acuity and the RCOphth NOD data (Table 4).

\section{Discussion}

This single-centre study provides a high-quality data set of $>5000$ consecutive cataract operations from a new ophthalmology unit. The completeness of these data compares favourably with previous reports using data from EMR in
Table 2 Post-operative complications compared to RCOphth NOD 2017

\begin{tabular}{|c|c|c|}
\hline $\begin{array}{l}\text { Presence or absence } \\
\text { of post-operative } \\
\text { complications }\end{array}$ & $\begin{array}{l}\text { Recorded in } \\
4938 \text { of eyes } \\
(98.6 \%), n(\%)\end{array}$ & $\begin{array}{l}\text { RCOphth NOD } 2017 \text { fo } \\
\text { operations with recorded } \\
\text { data ( } 35.6 \%), \%\end{array}$ \\
\hline $\begin{array}{l}\text { No post-operative } \\
\text { complications }\end{array}$ & $4454(90.2)$ & 88.6 \\
\hline $\begin{array}{l}\text { One or more post-operative } \\
\text { complications }\end{array}$ & $484(9.8)$ & 11.4 \\
\hline Post-operative uveitis & $129(2.6)$ & 3.2 \\
\hline Cystoid macular oedema & $99(2.0)$ & 2.7 \\
\hline $\begin{array}{l}\text { Corneal oedema/striae/ } \\
\text { Descemet's folds }\end{array}$ & $78(1.6)$ & 2.7 \\
\hline Raised IOP (>21 mm Hg) & $60(1.2)$ & 1.6 \\
\hline Reduction in vision ${ }^{\mathrm{a}}$ & $50(1.0)$ & \\
\hline Macular oedema & $33(0.7)$ & 0.0 \\
\hline Corneal decompensation & $17(0.3)$ & 0.2 \\
\hline Unexpected refractive outcome & $14(0.3)$ & 0.2 \\
\hline Vitreous in $\mathrm{AC}$ & $9(0.2)$ & 0.3 \\
\hline Leaking wound (Seidel +ve) & $7(0.1)$ & $<0.1$ \\
\hline Hypotony $<5$ & $6(0.1)$ & $<0.1$ \\
\hline Retained soft lens matter & $6(0.1)$ & 0.4 \\
\hline IOL decentred & $5(0.1)$ & 0.2 \\
\hline Iris to wound & $5(0.1)$ & $<0.1$ \\
\hline Vitreous to the section & $4(0.1)$ & 0.1 \\
\hline $\begin{array}{l}\text { Choroidal effusion/ } \\
\text { haemorrhage }\end{array}$ & $3(0.1)$ & $<0.1$ \\
\hline Retinal tear & $3(0.1)$ & $<0.1$ \\
\hline $\begin{array}{l}\text { Posterior capsule opacification } \\
\text {-YAG indicated }\end{array}$ & $3(0.1)$ & 0.1 \\
\hline Corneal epithelial defect & $2(<0.1)$ & $<0.1$ \\
\hline Endophthalmitis & $2(<0.1)$ & $<0.1$ \\
\hline Hyphaema & $2(<0.1)$ & $<0.1$ \\
\hline Post-operative eyelid oedema & $2(<0.1)$ & $<0.1$ \\
\hline Anterior capsulophimosis & $1(<0.1)$ & $<0.1$ \\
\hline Diplopia & $1(<0.1)$ & $<0.1$ \\
\hline Iris prolapse & $1(<0.1)$ & $<0.1$ \\
\hline Post-operative ptosis & $1(<0.1)$ & $<0.1$ \\
\hline Posterior capsule opacification & $1(<0.1)$ & 0.3 \\
\hline $\begin{array}{l}\text { Progression of diabetic } \\
\text { retinopathy }\end{array}$ & $1(<0.1)$ & $<0.1$ \\
\hline Retinal detachment & $1(<0.1)$ & $<0.1$ \\
\hline Vitreous haemorrhage & $1(<0.1)$ & $<0.1$ \\
\hline Other & $69(1.4)$ & 1.3 \\
\hline
\end{tabular}

$\mathrm{AC}$ anterior chamber, $I O L$ intraocular lens, $I O P$ intraocular pressure, RCOphth NOD Royal College of Ophthalmologists' National Ophthalmology Database

${ }^{a}$ Note reduction of vision was reported by the clinician using electronic medical record and is not the same as the RCOphth NOD definition of doubling of the visual angle or worse. We report a $1.26 \%$ rate of reduction in vision according to the RCOphth NOD criteria

the UK, not least because this data set includes $100 \%$ of the cataract operations performed in our unit within these dates. In the RCOphth NOD Audit Report 2017, no pre-operative VA data were recorded in $19.5 \%$ of cases and no post-operative complication data were recorded in $64.4 \%$. Pre- and post-operative VA data were recorded in only $57.1 \%$ of cases [5]. Incompleteness of VA 
Table 3 Post-operative visual acuity (VA) by pre-operative VA, intraoperative complications and posterior capsular rupture (PCR) for cases where pre-operative and post-operative VA are recorded

\begin{tabular}{llll} 
Percentages $(N)$ & \multicolumn{3}{l}{ Post-operative Snellen visual acuity } \\
\cline { 2 - 4 } All eyes (4906) & $\leq 6 / 6$ & $\leq 6 / 12$ & $\leq 6 / 24$ \\
& $40.8(2004)$ & $90.7(4449)$ & $96.8(4750)$
\end{tabular}

Presenting Snellen VA

$\leq 6 / 6 \quad 2.8 \%(137) \quad 70.8(97) \quad 99.3(136) \quad 100(137)$

$\leq 6 / 12 \quad 36.2 \%(1778) \quad 49.7(883) \quad 98.0(1743) \quad 99.8(1774)$

$\leq 6 / 24 \quad 67.6 \%(3316) \quad 43.0(1425) \quad 94.5(3134) \quad 99.1(3287)$

Intra-operative complications

$\begin{array}{lllll}\text { No } & 97.6 \%(4789) & 41.2(1972) & 90.9(4354) & 96.9(4642)\end{array}$

Yes $\quad 2.38 \%(117) \quad 27.4(32) \quad 81.2(95) \quad 92.3(108)$

PCR (RCOphth NOD definition)

$\begin{array}{lllll}\text { No } & 98.9 \%(4850) & 41.0(1989) & 90.8(4405) & 96.9(4702)\end{array}$

Yes $\quad 1.14 \%(56) \quad 26.8(15) \quad 78.6(44) \quad 85.7(48)$

RCOphth NOD Royal College of Ophthalmologists' National Ophthalmology Database

Table 4 Post-operative visual acuity in different post-operative time brackets and compared with RCOphth NOD benchmarks from 2017

\begin{tabular}{llll}
\hline & $\begin{array}{l}\text { Cases with } \\
\text { visual acuity } \\
\text { measurement } \\
\text { within 14 days } \\
\text { to 4 months } \\
\text { post-operative }\end{array}$ & $\begin{array}{l}\text { Cases with } \\
\text { visual acuity } \\
\text { measurement } \\
\text { within 1 day } \\
\text { to 6 months } \\
\text { post-operative }\end{array}$ & $\begin{array}{l}\text { RCOphth NOD } \\
\text { Benchmarks } \\
\text { (using 14 days } \\
\text { to 4 months) }\end{array}$ \\
\hline $\begin{array}{l}\text { Percentage } \\
\text { of eyes with } \\
\text { pre- and post- }\end{array}$ & $83 \%$ & $98 \%$ & \\
$\begin{array}{l}\text { operative data } \\
\text { in our cohort }\end{array}$ & & & \\
$\leq 6 / 6$ & $39.1 \%$ & $40.8 \%$ & $39 \%$ \\
$\leq 6 / 12$ & $89.9 \%$ & $90.7 \%$ & $88.6 \%$ \\
$\leq 6 / 24$ & $96.5 \%$ & $96.8 \%$ & $95.9 \%$
\end{tabular}

RCOphth NOD Royal College of Ophthalmologists' National Ophthalmology Database

data has been a historical problem in national data sets in the UK $[1,2,7]$ and the RCOphth NOD audit report 2017 acknowledges that 'completeness of pre-operative VA and post-operative VA outcome remain variable and an area for improvement in many centres'. We anticipate that the increasing adoption of paperless EMR will bring about this improvement. In the meantime, our $(98 \%$ complete) data appear to validate the benchmarks for VA outcomes reported in the RCOphth NOD audit reports. One way of improving the completeness of VA outcome would be to include data on all patients. This would require a change in the time-based definition of post-operative VA defined by RCOphth NOD. We note an approximate $15 \%$ loss of post-operative VA data in our cases when adhering to RCOphth NOD criteria for reporting post-operative VA. Our data suggest that including data on all patients would not materially change the VA outcomes.

When analysing intra-operative complications, this study found a PCR rate of $1.14 \%$, which compares well with the 1.5 and $1.8 \%$ PCR rate from RCOphth NOD 2017 and 2016, respectively. Of note, our data set is comprehensive and we have not excluded cataract cases that RCOphth NOD defines as ineligible in its statistical analysis plan. The overall rate of post-operative complication was $9.8 \%$ in this study with post-operative uveitis and corneal oedema accounting for $4.2 \%$. Although our rate of major intra-operative complication (PCR) was lower than that recorded in the RCOphth NOD, our rate of recorded post-operative less serious complications $(9.8 \%)$ was higher than the 5.8\% reported in RCOphth NOD 2016 and lower than the 11.4\% reported in RCOphth NOD 2017. These differences between our results and those of the RCOphth NOD and between successive RCOphth NOD reports raise an interesting issue about the definition of complications and recording in electronic records. At the first post-operative review, our electronic record forces documentation of the presence or absence of the findings listed in Table 2. Corneal oedema and post-operative uveitis, for example, are present in almost all patients at some point after cataract surgery and whether these are recorded as a complication depends both on the timing of post-operative review and the ability or inclination of the clinician reviewing the patient to distinguish between complication and normal post-operative course. Many of our patients were reviewed at 1-2 weeks post-surgery rather than the usual 3 weeks and this may account for some of the reported cases of corneal oedema and post-operative uveitis. Similarly, recorded rates of cystoid macular oedema will depend on whether patients have post-operative optical coherence tomographic scans of the retina and whether cystoid macular oedema is defined clinically or tomographically. In order to accurately benchmark rates of post-operative complications, these complications need to be defined.

In some units in the UK, patients are followed up by community opticians and not seen by the operating unit post-operatively. This is a further reason for loss of electronic data during the cataract pathway. Our centre invites all of our patients to attend for post-operative review after cataract surgery and records data exclusively electronically. Hence we have been able to record follow-up in $98.6 \%$ of operations and record post-operative VA in $98.0 \%$ of operations. This represents an almost complete data set. We attribute the small data loss to non-attendance for follow-up, inability to record VA (learning difficulties/ 
cognitive impairment) and human error in neglecting or forgetting to enter data. In some fields, we have recorded $100 \%$ data completeness. This is usually because the EMR forces the user to make an entry for this field. Forced choice data entry leads to high levels of data completeness but not necessarily data accuracy. One of the forced choice data fields in Medisoft Ophthalmology is the presence or absence of ocular co-pathology. An answer was recorded in $100 \%$ of cases, but in our cohort, co-pathology was recorded as present in just $29.7 \%$ of cases compared with $46.7 \%$ of cases in the RCOphth NOD audit report 2017. Our cataract patient cohort is comprehensive containing both new referrals and patients who already attend the clinic with other eye conditions, so we were surprised to see the relatively low level of recorded ocular co-pathology. One explanation for this is that our cohort does in fact contain a lower proportion of patients with ocular co-pathology compared to the RCOphth NOD audit. Another explanation is that we have not recorded the presence of co-pathology accurately in our patients. This raises an important issue in paperless systems: In order to enter data in mandatory fields faithfully, those data must be easily accessible while the field is being filled. In our software, it is difficult to access the past ophthalmic history and findings while completing the operation note. This barrier may explain the tendency for surgeons to tick the "no ocular co-pathology" mandatory field when filling the operation note in order to maintain efficiency in the operating theatre.

The easy availability of high-quality fully representative outcome data is just one benefit of the move to paperless record-keeping. It provides real-time feedback and the ability to audit results rapidly and comprehensively and then instigate improvements. However, data will always be limited to accurate record keeping by the clinician regardless of how it is recorded.

Our study represents one of the most comprehensive and complete data sets on cataract surgery to be reported and appears to validate the outcome benchmarks reported by the RCOphth NOD audit reports.

\section{Summary}

\section{What was known before}

- Large data sets on cataract outcomes are limited by incomplete collection of key primary outcome indicators, which in turn may affect the quality of the data.

\section{What this study adds}

- This study demonstrates that fully paperless ophthalmology units can be achieved in the NHS and that these have the ability to produce comprehensive cataract surgery outcome data.

- The comprehensiveness of the data and the absence of selection bias mean that these data can be used with confidence in benchmarking and audit.

Acknowledgements The authors would like to thank Mr. Paul Donachie for his comments and editing of the final manuscript.

\section{Compliance with ethical standards}

Conflict of interest The authors declare that they have no conflict of interest.

Publisher's note: Springer Nature remains neutral with regard to jurisdictional claims in published maps and institutional affiliations.

\section{References}

1. Day AC, Donachie PH, Sparrow JM, Johnston RL, Royal College of Ophthalmologists' National Ophthalmology Database. The Royal College of Ophthalmologists' National Ophthalmology Database study of cataract surgery: report 1, visual outcomes and complications. Eye (Lond). 2015;29:552-60.

2. Donachie P, Sparrow JM, Johnston RL. National Ophthalmology Database Audit. The Royal College of Ophthalmologists; 2016. Available at https://www.nodaudit.org.uk/u/docs/20/muedbbgqwv/ NOD\%20Audit\%20Annual\%20Report\%202016.pdf (accessed 10 February 2018)

3. DAMA UK. The six primary dimensions for data quality assessment. 2013. Available at http://www.damauk.org/RWFilePub.php? $\& \mathrm{cat}=403 \& \mathrm{dx}=2 \& \mathrm{ob}=3 \& \mathrm{rpn}=$ catviewleafpublic $403 \& \mathrm{id}=106193$ (accessed 10 February 2018)

4. Lundström M, Barry P, Henry Y, Rosen P, Stenevi U. Evidencebased guidelines for cataract surgery: guidelines based on data in the European Registry of Quality Outcomes for Cataract and Refractive Surgery database. J Cataract Refract Surg. 2012;38:1086-93.

5. Donachie PHJ, Sparrow JM. National Ophthalmology Database Audit. The Royal College of Ophthalmologists; 2017. Available at https://www.nodaudit.org.uk/u/docs/20/ughyyctynz/NOD\%20Audit \%20Annual\%20Report\%202017.pdf (accessed 10 February 2018)

6. National Advisory Group on Health Information Technology in England. Making it work: harnessing the power of health information technology to improve care in England. 2016. Available at https://www.gov.uk/government/uploads/system/uploads/atta chment_data/file/550866/Wachter_Review_Accessible.pdf (accessed 10 February 2018)

7. Sparrow JM, Taylor H, Qureshi K, Smith R, Birnie K, Johnston $\mathrm{RL}$, et al. The Cataract National Dataset electronic multi-centre audit of 55,567 operations: risk indicators for monocular visual acuity outcomes. Eye (Lond). 2012;26:821-6. 